\title{
Article \\ Analyses of Contact Networks of Community Dogs on a University Campus in Nakhon Pathom, Thailand
}

\author{
Tipsarp Kittisiam ${ }^{1,2, * \mathbb{C}}$, Waraphon Phimpraphai ${ }^{1, *}$, Suwicha Kasemsuwan ${ }^{1} \mathbb{C}$ and Krishna Kumar Thakur ${ }^{2} \mathbb{D}$ \\ 1 Department of Veterinary Public Health, Faculty of Veterinary Medicine, Kasetsart University, \\ Kamphaeng Saen, Nakhon Pathom 73140, Thailand; fvetswk@ku.ac.th \\ 2 Department of Health Management, Atlantic Veterinary College, University of Prince Edward Island, \\ Charlottetown, PE C1A 4P3, Canada; kthakur@upei.ca \\ * Correspondence: tipsarp.k@gmail.com (T.K.); fvetwrp@ku.ac.th (W.P.)
}

check for updates

Citation: Kittisiam, T.; Phimpraphai, W.; Kasemsuwan, S.; Thakur, K.K. Analyses of Contact Networks of Community Dogs on a University Campus in Nakhon Pathom, Thailand. Vet. Sci. 2021, 8 , 299. https://doi.org/10.3390/ vetsci8120299

Academic Editor:

Alessandra Pelagalli

Received: 28 October 2021

Accepted: 26 November 2021

Published: 30 November 2021

Publisher's Note: MDPI stays neutral with regard to jurisdictional claims in published maps and institutional affiliations.

Copyright: (c) 2021 by the authors. Licensee MDPI, Basel, Switzerland. This article is an open access article distributed under the terms and conditions of the Creative Commons Attribution (CC BY) license (https:/ / creativecommons.org/licenses/by/ $4.0 /)$.

\begin{abstract}
Free-roaming dogs have been identified as an important reservoir of rabies in many countries including Thailand. There is a need for novel insights to improve current rabies control strategies in these countries. Network analysis is commonly used to study the interactions between individuals or organizations and has been applied in preventive veterinary medicine. However, contact networks of domestic free-roaming dogs are mostly unexplored. The objective of this study was to explore the contact network of free-roaming dogs residing on a university campus. Three one-mode networks were created using co-appearances of dogs as edges. A two-mode network was created by associating the dog with the pre-defined area it was seen in. The average number of contacts a dog had was 6.74. The normalized degree for the weekend network was significantly higher compared to the weekday network. All one-mode networks displayed small-world network characteristics. Most dogs were observed in only one area. The average number of dogs which shared an area was 8.67. In this study, we demonstrated the potential of observational methods to create networks of contacts. The network information acquired can be further used in network modeling and designing targeted disease control programs.
\end{abstract}

Keywords: free-roaming dogs; contact network; network analysis

\section{Introduction}

Free-roaming dogs include all unconfined dogs with varying relationships with humans. They can be grouped by their ownership status into three loose categories: owned dogs, semi-owned (which means the owner of the dog is not clearly defined, but the dog is associated with at least one human or household) or unowned dogs (sometimes referred to as feral dogs or stray dogs) [1]. Semi-owned dogs are also known as community dogs since they are related to multiple households in the community [2,3]. However, it is not always practical to correctly discern a dog from one subcategory to another without appropriate information. Free-roaming dogs can be found in multiple countries across Asia, including Indonesia (especially in Bali), the Philippines, India, Sri Lanka and Thailand [4]. In 2016, the Department of Livestock Development (DLD), Thailand, reported a total of 7.4 million dogs (6.6 million owned and over 700,000 unowned dogs). Dogs are commonly raised as free-ranging [5] and unowned dogs are often fed by people in the community [6]. Thus, free-roaming dogs can be found commonly in Thailand.

The potential public health and ecological health impacts from free-roaming dogs has led to developing various measures to control their population such as a capture-neuterrelease program. The presence of free-roaming dogs living near wildlife conservation areas introduces a concern for the ecological impact of dogs as an invasive alien species [7]. With regards to their importance in public health, free-roaming dogs have been identified as a reservoir for rabies in many endemic countries [8]. Rabies is a lethal zoonotic disease. The World Health Organization (WHO) has asserted the importance of controlling rabies in 
dogs, especially in countries with free-roaming dogs, in order to eliminate human rabies [9]. Rabies is endemic in Thailand. Despite the collaborative efforts between the municipalities and the DLD to control the disease, there are still reports of positive cases detected through passive surveillance which suggest potential outbreaks [10]. Suboptimal vaccine coverage has been identified as one of the major obstacles in controlling rabies in Thailand [11]. Adjunct interventions such as orally-administered rabies vaccinations are being developed to increase the total vaccine coverage [12]. Strategically vaccinating dogs with higher than average contacts in addition to increasing efforts to vaccinate the free-roaming dog population, could be an effective strategy in controlling rabies. Hence, leading to the sufficient control of rabies spread in free-roaming dogs and preventing the spillover to humans. This has encouraged the researchers to develop a method to help identify high priority targets for vaccination.

Network analysis is a commonly used method to study the relationships between individuals. It has been utilized to study the contact networks of humans $[13,14]$ and various animals, including lizards [15], dolphins [16] and non-human primates [17]. Furthermore, various networks of livestock movements such as cattle [18,19] and swine [20-22] have been studied in the interest of controlling disease spread. Network analysis is used in veterinary epidemiology to explain and model the relationship (such as contacts) between animals or group of animals based on movement, co-occurrence, proximity, etc., and the important role such contacts play in disease spread within and between groups [23-25].

Few studies describing contact networks of owned free-roaming dogs have been conducted. A study in Cheshire, UK [26], demonstrated the high level of potential contacts which could occur from dogs that share walking routes using a two-mode network. The observed network was highly connected and had small-world network characteristics that could support the fast spread of an infectious disease in the network [26]. Dog-to-dog networks were studied in Torres Strait, Australia [27] and in a separate study dog-to-dog networks in four countries (Chad, Guatemala, Indonesia and Uganda), were compared [28]. Both these studies also identified the small-world structure of the networks which could lead to rapid spread of disease. Spatial proximity was identified as an influential factor in the centrality of a dog in both studies $[27,28]$. Network-based simulation of rabies spread utilizing the network parameters $[29,30]$ emphasize the role of network analysis in understanding how a disease can potentially spread in these populations and identify individuals or groups that can be targeted for surveillance and disease control. To the best of our knowledge, there has not been a study conducted on unowned or semi-owned dogs in Thailand. The objective of this study is to describe the contact patterns between dogs and the areas which they frequent on a university campus in Thailand by using network analysis. Additionally, the study aims to describe the network between the free-roaming dogs observed on the campus, as well as to characterize the network properties of both the dog-to-dog network and dog-to-area network to identify key nodes that have a potential for disease spread.

\section{Materials and Methods}

\subsection{Study Site}

This study was conducted at Kasetsart University, Kamphaeng Saen Campus, which is located in Nakhon Pathom Province, in the Central Region of Thailand. The campus area is estimated to be over 3000 acres, most of which are agricultural fields. The campus has around 2000 staff and 14,600 students [31]. Free-roaming dogs are commonly found on campus. In a previous study [32], the campus area was divided into 96 polygons using roads and natural boundaries such as lakes in order to estimate the population of free-roaming dogs within the campus. For the purpose of this study, the 96 polygons were categorized into urban and rural types using two main criteria: assumed human activity and occupancy of human-made structures (not including plantations) (Figure 1). Additional information regarding the categorization of urban and rural types can be found in Appendix A. 


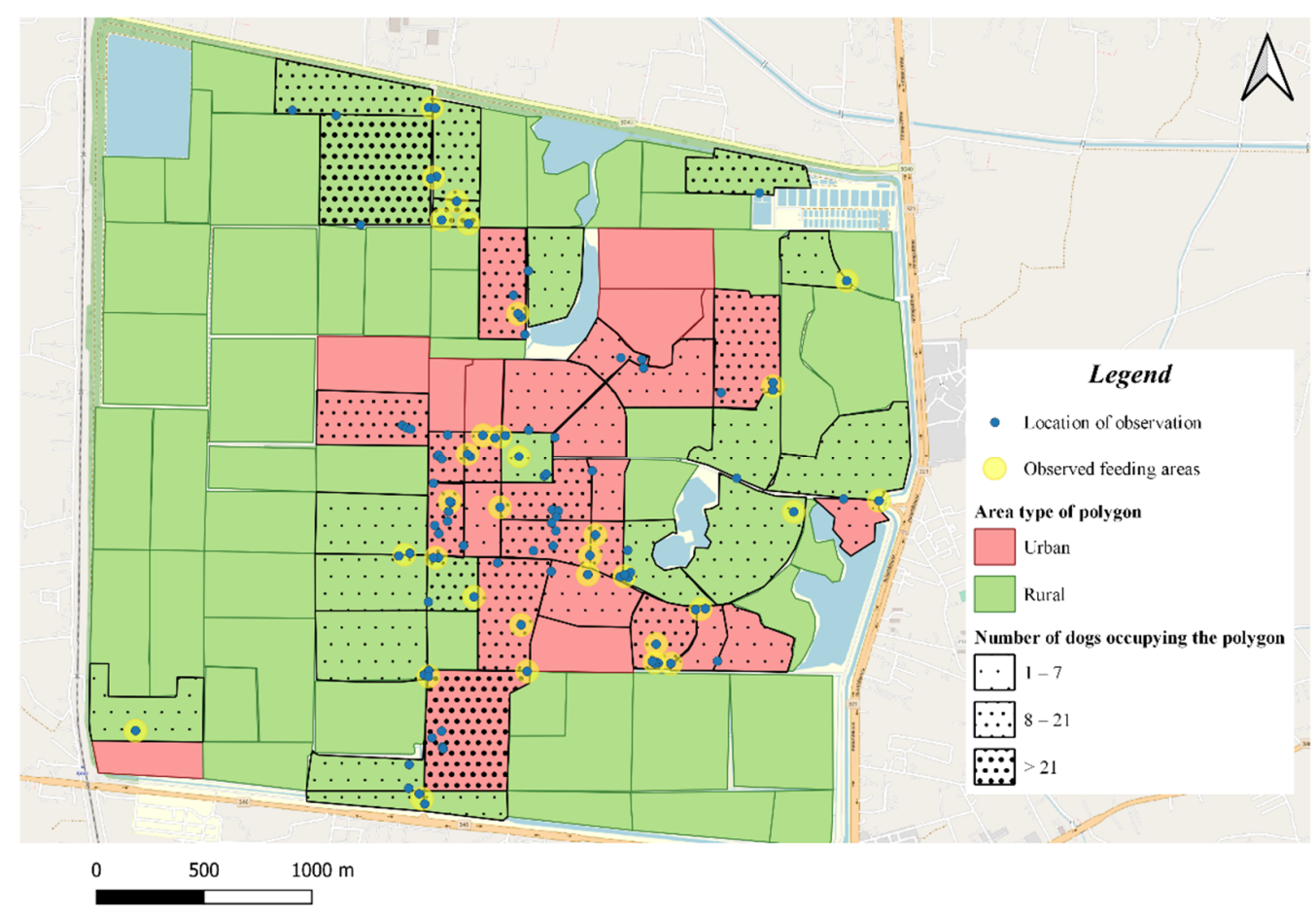

Figure 1. Map of Kasetsart University, Kamphaeng Saen Campus in 2020. The map of Kasetsart University, Kamphaeng Saen Campus was made using OpenStreetMap. The areas are defined as urban (pink) or rural (green), according to the level of human activity and the proportion of buildings in the polygon. The number of dogs occupying the polygon is depicted with the density of the black spots filled in the polygon. The locations of dogs observed in the study are displayed as blue triangular symbols on the map.

In the most recent survey, at least 584 dogs were estimated to live on campus in 2019 [32]. The surveyed population only included community dogs or semi-owned dogs. Dogs in the previous study are routinely fed by staff of the university on weekdays which suggests that working hours could affect the grouping behavior of the dogs.

\subsection{Data Collection}

Visual observations were conducted to record dog sightings and their contacts. They were conducted by one observer (TK). Caretakers of community dogs on campus regularly feed the dogs before (6-7 a.m.) or after work (4-6 p.m.). In order to increase the chances of capturing the largest number of dogs during a cross-sectional observation, the planned observations were conducted between these specific times. During the sighting of a dog, a photograph was taken by a digital camera or a mobile phone. The dog's overall physical appearance, such as the coat, coat color and obvious visible markings, were recorded along with its age, sex and castration status when possible. Neuter status was assumed from the absence of testes in male dogs. However, for female dogs, ovariohysterectomy does not leave a noticeable scar. Therefore, they are assumed to be intact unless the dog was previously branded after being spayed.

A dog's initial response to strangers can be helpful in evaluating a dog's overall attitude towards humans and assess the likelihood of biting humans [2]. The observer had no prior contact with the dogs in this study, therefore, can be considered a stranger to the dogs. A modified "approach test" [2] was used to document the vocalization, dismissive, fearful and friendly behavior from the dog. The photographs and records from each observation were used to associate the identity of each dog and their co-occurrences.

Results from the previous study [32] were used to determine the transect route of observations. GPS coordinates were collected at the location of the visual observations to associate the location of the group of dogs with the previously defined areas. The observer 
also documented evidence of caretakers in the area such as a feeding bowl or leftover dog food. The mobile application, Epicollect5 $[33,34]$ was used to facilitate the data collection and recording.

Observations were carried out on 16 different days across four months (September to November 2020). On average, 6-7 polygons were observed in one day. The average duration of one observation was $7 \mathrm{~min}$ and $31 \mathrm{~s}$ (range 1-28 min). Records of the observations along with the corresponding photos from different days were collated and examined to eliminate duplicate records of the same dog.

The present study was conducted during the COVID-19 pandemic, which meant the majority of classes were held online and students were off-campus. However, the faculty and staff were not required to work remotely. The amount of human activity, on campus, during the studied time should be similar to the amount of activity during summer and winter breaks.

\subsection{Data Analysis}

All the observation data were collected using Epicollect5, which was later imported and analyzed using R (Version 4.0.3) [35]. After the identity of each dog was verified, the data were transformed into a node list. The attributes (age, sex, castration status and behavior towards humans) were also included in the node list. An edge list of cooccurrences between dogs was created from the list of dogs encountered during each observation. Co-occurrence is defined as the presence of dogs in the same polygon within the same observation period. The node and edge lists were then utilized to create the onemode (dog-to-dog) network. For the two-mode (dog-to-polygon) network, the edge list was created by relating the location of the dog to the polygon by which it was observed, using QGIS (Version 3.10.11) [36,37]. The node list of polygons included attributes regarding the possible food source and type of area (urban or rural). The polygon node list and dog node list were combined into the two-mode node list in order to create the two-mode network.

The network analysis software, UCINET (Version 6.720) [38] and Gephi (Version 0.9.2) [39] were used to create and visualize an overall dog-to-dog network (for the entire study duration) of all the dogs observed during the same observation period from the node and edge list described earlier. The grouping behavior of dogs is suspected to be influenced by the amount of human activity on campus and their feeder, which are only active during the weekdays. Therefore, two networks were created separately for dogs observed on the weekdays and weekends. In addition, the dog-to-polygon network was created using the previously described two-mode node and edge lists to describe the assumed association of the dog with the areas of interest.

Network statistics were computed for all one-mode networks, including network diameter, average path length and density. Components within the networks were identified and fragmentation of the network was calculated. Degree centrality and normalized degree were computed for each network. Statistical tests with permutation-based significances provided in UCINET were used to compare normalized degrees between different networks or groups within a network.

The main component is the largest connected group of nodes in the network. Normalized betweenness (nBetweenness) and normalized closeness (nCloseness) were computed from the main component of each network. The cut-points of the main component from the overall dog-to-dog network were identified using NetDraw (Version 2.175) [40]. Furthermore, the cut-points were removed and sub-groups were identified within the main component.

In addition, for each of the one-mode networks (overall, weekday and weekend), five hundred random networks of equal size and density were generated. The average path length $(\mathrm{L})$ and average clustering coefficients $(\mathrm{C})$ of the random networks of the same size and density were calculated and compared with the observed network to determine whether it had a small-world structure. Small-world networks have a higher clustering coefficient and lower average path length compared to the random networks. Furthermore, 
an alternative and simplified approach was also used to assess the small-world-ness (S) of a network as proposed by Humphries et al. [41,42] using the following formula:

$$
\mathrm{S}=\left(\mathrm{C}_{\text {observed }} / \mathrm{C}_{\text {random }}\right) /\left(\mathrm{L}_{\text {observed }} / \mathrm{L}_{\text {random }}\right)
$$

Networks that have $S>1$ are defined as small-world networks.

Network statistics computed for the two-mode network include the density, transitivity, fragmentation and average path length. Two-mode centrality indices calculated include the average degree, nBetweenness and nCloseness. The two-mode degrees were further analyzed using statistical tests with permutation-based significances in UCINET.

A permutation-based simple linear regression model was used to determine the relationship between the normalized degree of dog nodes, calculated from the two-mode network and the nBetweenness, calculated from the main component of the overall dogto-dog network. The model was fit using the normalized degree of dog nodes to predict the nBetweenness.

\section{Results}

\subsection{Descriptive Analysis}

A total of 261 dogs were recorded and identified from 113 observations during the study period at various locations on campus. Fifty-one observations were conducted during weekdays and 62 observations were conducted on the weekends. The observed areas extended into 42 polygons, of which 20 and 22 polygons were classified as urban and rural areas, respectively. One hundred and twelve dogs were observed only on weekdays, 80 were observed only on weekends and 69 were observed on both weekdays and weekends. The majority of dogs identified were adults $(247 / 261)$ and only a few puppies were spotted (14/261). There were 101 female and 90 male dogs identified, 70 dogs could not be categorized for sex. Only 5\% (5/102) of females were spayed and $11 \%(9 / 81)$ of males were neutered. The most exhibited behavior towards a human stranger during the first encounter was fear $(131 / 261)$, followed by disregard $(64 / 261)$, friendly behavior $(52 / 261)$ and barking $(14 / 261)$ (Table 1$)$.

Table 1. Descriptive characteristics of free-roaming dogs observed in Kasetsart University, Kamphaeng Saen.

\begin{tabular}{lccc}
\hline & Characteristics of Dogs & Number of Dogs & Percentage \\
\hline & Age & 247 & \\
Adult & & 14 & 94.64 \\
Juvenile & Sex & & \\
\hline & & 96 & 36.78 \\
Female & 81 & 31.03 \\
Male & 5 & 1.92 \\
Spayed females & 9 & 3.45 \\
Neutered males & 70 & 26.82 \\
Unknown & & \\
\hline Behavior towards human during the first occurrence & 14 & 5.36 \\
Bark & 64 & 24.52 \\
Dismissive & 131 & 50.19 \\
Fearful & 52 & 19.92 \\
Friendly & Total & 261 & 100 \\
\hline
\end{tabular}

\subsection{One-Mode Networks (Dog-to-Dog Networks)}

The overall dog-to-dog network (Figure 2) contained a total of 261 nodes (dogs) and 1760 edges (co-occurrences). The average degree was 6.74 (range 0-29). Over fifty percent of all observed dogs each had degree $\leq 5(150 / 261)$ and only $3.83 \%(10 / 261)$ had 
degrees over 20. Additional measures of centrality are displayed in Table 2. Fifty-four components were identified in the network. There were 20 isolated nodes. The largest three components contained $117(45 \%), 13(5 \%)$ and $8(3 \%)$ dogs, respectively. The component ratio is calculated from the number of components minus one divided by the number of nodes minus one.

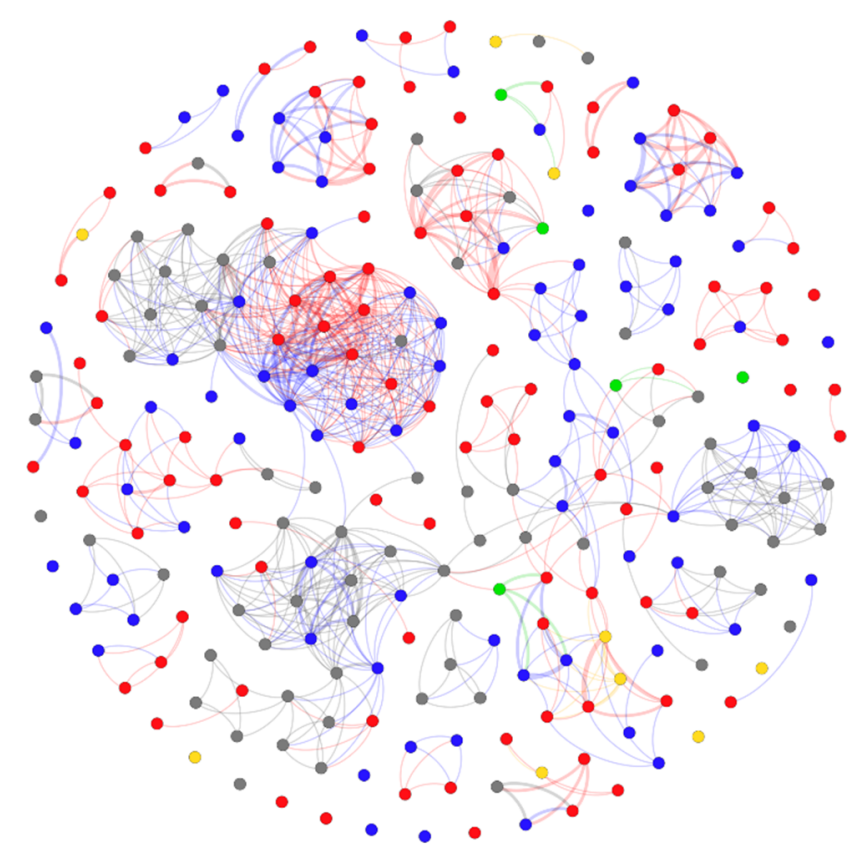

Figure 2. The overall dog-to-dog network graph, created in Gephi. The nodes are free-roaming dogs observed during study in Kasetsart University, Kamphaeng Saen, in 2020. The edges represent the co-occurrences of the dogs. The colors of the nodes represent the sex and castration status of each dog: male (blue), female (red), neutered male (yellow), spayed female (green) and unknown (grey).

Table 2. Network characteristics of the dog-to-dog networks observed in Kasetsart University, Kamphaeng Saen Campus.

\begin{tabular}{cccc}
\hline Network Characteristics & Overall Dog-to-Dog Network & Weekday Dog-to-Dog Network & Weekend Dog-to-Dog Network \\
\hline Total number of nodes & 261 & 181 & 149 \\
\hline Total number of edges & 1760 & 1066 & 902 \\
\hline Average Degree & 6.743 & 5.890 & 6.054 \\
\hline Density & 0.026 & 0.033 & 0.041 \\
\hline Average Distance (SD) & $5.390(3.358)$ & $1.084(0.324)$ & $3.304(1.601)$ \\
\hline Diameter & 14 & 3 & 7 \\
\hline nBetweenness (SD) ${ }^{1}$ & $3.953(9.692)$ & $0(0)$ & $3.819(10.249)$ \\
\hline nCloseness (SD) ${ }^{1}$ & $18.952(3.834)$ & $100(0)$ & $29.931(5.347)$ \\
\hline Small-world-ness (S) & 25.148 & 142.168 & 28.706 \\
\hline Components & 54 & 50 & 33 \\
\hline Component Ratio & 0.204 & 0.272 & 0.216 \\
\hline Size of largest component & 117 & 20 & 66 \\
\hline Fragmentation & 0.792 & 0.974 & 0.793 \\
\hline
\end{tabular}

\footnotetext{
${ }^{1}$ The nBetweenness and nCloseness were calculated from the largest component.
}

The overall dog-to-dog network had a high clustering coefficient (1.131) compared to the average clustering coefficient calculated from the 500 random networks $(0.026)$. Furthermore, the $\mathrm{S}$ computed from the average path length and clustering coefficient was 
25.148, which further confirmed that this network exhibited characteristics of a small-world network $(S>1)$.

Permutation-based ANOVA was performed to assess the difference between normalized degree distribution of studied dogs based on their sex and neuter status. However, no statistical difference was observed ( $p$-value $>0.05, \mathrm{~F}=1.849, \mathrm{df}=4)($ Figure 3A) (Table S1). Cut-points within the largest component were generated in NetDraw. Removal of the cutpoints in the main component created 10 smaller components and two isolates (Figure S1).
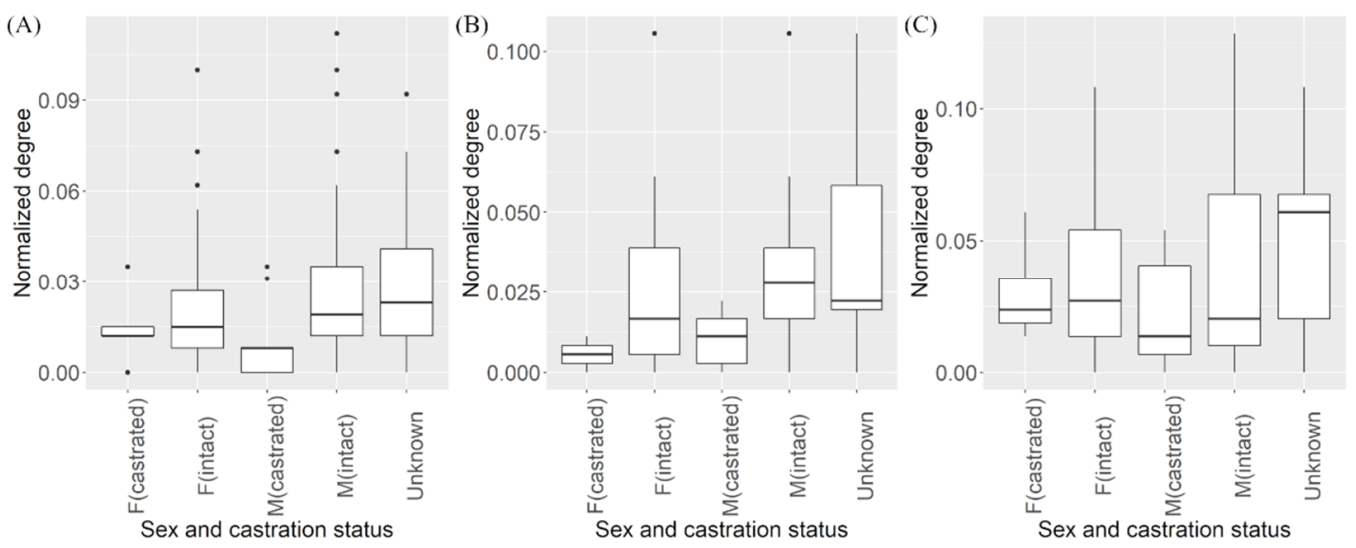

Figure 3. Plots of the normalized degrees stratified by the sex and castration status. Distribution of normalized degrees calculated from the (A) overall, (B) weekday and (C) weekend dog-to-dog networks observed in Kasetsart University, Kamphaeng Saen in 2020. The dots represent extreme values in the dataset.

\subsection{Comparison between Weekday-Weekend One-Mode Networks}

Separate one-mode networks were created for dogs encountered during the weekdays and weekends to assess the effect of human activity on campus on the grouping behavior of dogs. The network of dogs observed on weekdays had 181 nodes and 1066 edges with an average degree of 5.89. The network was highly fragmented (97.4\%), with a total of 50 components and had small-world topology. The largest component included 20 nodes or $11 \%$ of the total number of nodes. The clustering coefficient for the weekday network was 1.05 and the average path length was 1.08 .

The network of dogs observed on the weekends had similar characteristics. The clustering coefficient was 1.05 and the average path length was 3.30, consistent with the small-world network topology. The weekend network had only 149 nodes and 902 edges with an average degree of 6.05. It was also highly fragmented (79.3\%), having fewer components (33) compared to the weekday network. The largest component contained 66 nodes or $44 \%$ of the total number of nodes. The permutation-based t-test showed the normalized degree of dogs' contacts during the weekends (average $=0.041$ ) was significantly higher than on the weekdays $(0.033)(p$-value $=0.01$, mean difference $=0.008)($ Figure $3 B, C)$ (Table S1).

\subsection{Two-Mode Network (Dog-to-Polygon Network)}

A two-mode network (Figure 4) was created to visualize and quantify the overlapping area-use between dogs. The network had 303 nodes and 364 edges in total. The average degree of dog nodes, which indicates the number of polygons it appears in, was 1.40 (range 1-4). The average degree for the polygon, representing the total number of dogs observed in each polygon, was 8.67 (range 1-38). A summary of network measures from the dog-to-polygon network is displayed in Table 3. The normalized degrees of urban and rural area nodes (polygons) were compared using a permutation-based $t$-test (Table S1). However, no significant difference between the number of dogs associated with each area type was detected ( $p$-value $>0.05$ ) (Figure 5A). 


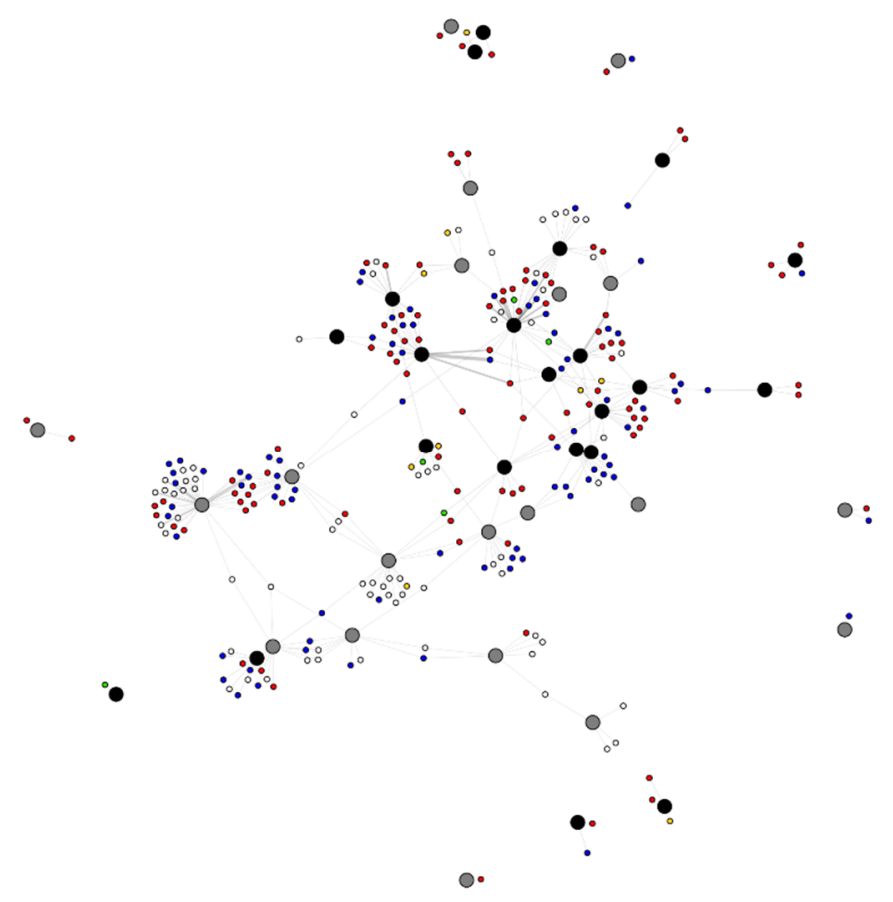

Figure 4. The two-mode dog-to-polygon network graph studied in Kasetsart University, Kamphaeng Saen in 2020, created in Gephi. The large circular nodes represent polygons. They were classified as urban (black) and rural (grey), according to buildings and human activity in the area. The small circular nodes represent dogs according to the sex and castration status: male (blue), female (red), neutered male (yellow), spayed female (green) and unknown (white).
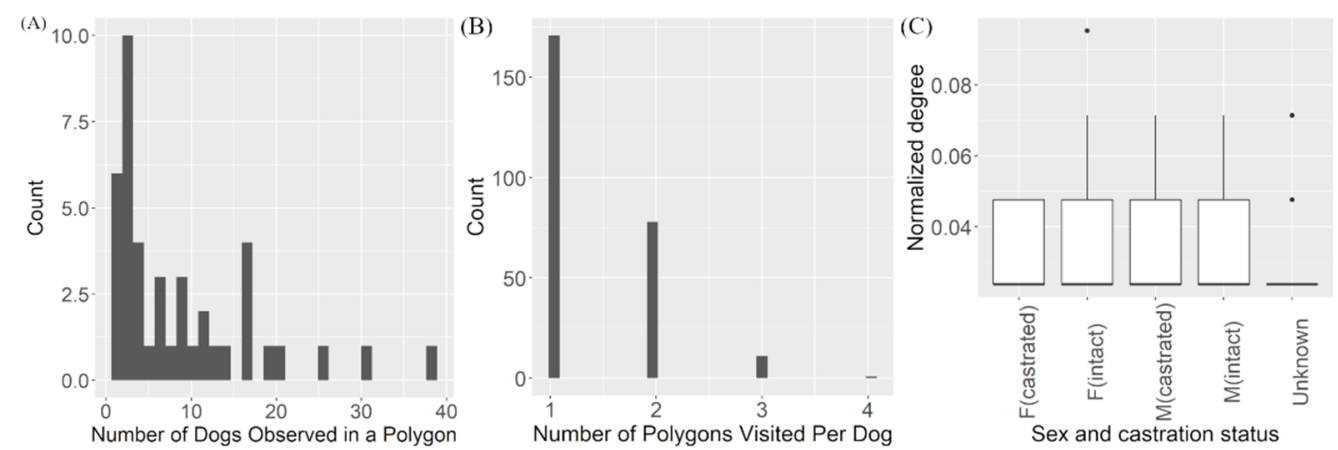

Figure 5. Histograms depicting the (A) degree of polygon nodes and (B) degree of dog nodes calculated from the two-mode network and the (C) box-plot of normalized degrees of dog nodes according to sex and castration status. The two-mode dog-to-polygon network measures included the degrees and normalized degrees of the two respective modes. Histograms depicting (A) the degree of polygon nodes (can be interpreted as the number of dogs observed in the same polygon (B) the degree of dog nodes (the number of polygons an individual dog was associated with) and (C) Box-plots showing the distribution of normalized degrees calculated from dog nodes was used to visualize the average number of polygons visited according to the sex and castration status. The dots on the plot represent extreme values.

A significant association was found between the normalized two-mode degree and nBetweenness of dog nodes in the main component ( $p$-value $=0.01, \mathrm{~F}(46,1)=7.102$, R-square $=0.134$ ) by using a permutation-based simple linear regression model (Table S1). 
Table 3. Network characteristics of the two-mode dog-to-polygon networks observed in Kasetsart University, Kamphaeng Saen Campus.

\begin{tabular}{|c|c|}
\hline Network Characteristics & Value \\
\hline Number of total nodes & 303 \\
\hline Number of total edges & 364 \\
\hline Density & 0.033 \\
\hline Transitivity & 0.698 \\
\hline Fragmentation & 0.235 \\
\hline Average Distance & 5.706 \\
\hline Diameter & 14 \\
\hline Norm Distance & 0.403 \\
\hline \multirow[t]{2}{*}{ Radius } & 1 \\
\hline & Dog nodes \\
\hline Number of dog nodes & 261 \\
\hline nDegree 1 & 0.033 \\
\hline Average Degree $(\min -\max )^{1}$ & $1.395(1-4)$ \\
\hline Average nBetweenness ${ }^{1}$ & 0.006 \\
\hline \multirow[t]{2}{*}{ Average nCloseness ${ }^{1}$} & 0.259 \\
\hline & Polygon nodes \\
\hline Number of polygon nodes & 42 \\
\hline nDegree $^{1}$ & 0.033 \\
\hline Average Degree $(\min -\max )^{1}$ & $8.667(1-38)$ \\
\hline Average nBetweenness ${ }^{1}$ & 0.05 \\
\hline Average nCloseness ${ }^{1}$ & 0.142 \\
\hline
\end{tabular}

\section{Discussion}

The networks of free-roaming dogs in Kasetsart University, Kamphaeng Saen Campus, Thailand were created and characterized in this study. This is likely the first study in Thailand to establish contact patterns of community dogs inhabiting a shared space. The computed degree centrality from the overall network represented the contacts between each pair of dogs. Small-world characteristics were observed in the studied networks. In addition, we also identified potential cut-points in the network based on their betweenness, which can be applied to target interventions and formulate specific disease mitigation strategies. Dogs with high risk of disease spread can be assumed using the identified network measures such as high degree and high betweenness. For example, a dog with high risk of disease spread can be prioritized for vaccination or captured, to minimize the further spread within the connected network. Network modelling can also be used to predict the potential disease spread and evaluate the efficacy of the vaccination.

In a previous study of free-roaming dog networks in Torres Strait, Australia [27], three networks were created, one from each community. The networks were well connected. In two networks (out of three), all dogs were connected within a single component. The small-world structure was observed in all three networks. Similarly, the networks of owned free-roaming dogs in Chad, Guatemala, Indonesia and some sites in Uganda [28] were also well connected and displayed small-world characteristics. The observed dogto-dog networks in our study also demonstrated a high clustering coefficient and short average path length, characteristics consistent with small-world topology. Although the average path length is longer when compared to the random networks, in smaller networks (200-3000 nodes), having a higher clustering coefficient is indicative of being a small-world network [42,43]. Furthermore, we also computed the network small-world-ness (S) value to confirm this finding. In small-world networks, an infectious disease can spread quickly within clusters and reach distant individuals within the network in a small number of steps $[44,45]$.

The distribution of degree in the dog-to-dog network, including the weekday and weekend subsets, showed similar right-skewed distribution to the degree observed in 
the networks of dogs in previous studies $[27,28]$. These authors reported that dogs were generally associated with a few dogs for long durations while larger gatherings occur in shorter periods of time [27]. This is consistent with the findings in our study. No significant difference was observed when comparing the normalized degrees of dogs with different sex and castrations status in the dog-to-dog network and the dog-to-polygon network. Therefore, we could not conclude whether there was an influence of dog's gender on clustering and diffused movement.

The lower degrees observed during the weekdays compared to the weekends could reflect the effect of external factors, such as the presence of people and automobile traffic, which affect group dispersal [46]. Conversely, considerably less human activity and traffic are seen on campus on the weekends, resulting in larger groups observed. This finding was initially thought to be due to the result of human-shy dogs appearing in the weekend groups. However, the proportion of the groups stratified by behavior showed the number of fearful dogs was equally high in both weekday and weekend networks (Figure S2). It is more likely that the dogs tend to gather in smaller groups in the presence of humans regardless of its' familiarity with people. This has been previously discussed as a possible consequence of larger groups attracting unwanted attention [46]. Therefore, it is naturally more beneficial for the dogs to remain as a small group in the presence of humans. Another possible explanation could be related to the absence of a food source. The lack of a feeder or human refuse during the weekend could motivate the dogs to aggregate and roam further in search of food [47]. This was also observed during previous studies on campus [32]. Our finding of increased degree centrality in the absence of humans is in agreement with previous studies which observed the roaming activity of dogs to be associated with human activity [48]. Moreover, this association should be further investigated to see the extent of its' effect and explore the possible uses in disease control planning. For example, there could be substantially larger gatherings during summer or winter breaks. Therefore, vaccination or capture-neuter-release programs may be planned accordingly.

Human activity has been shown to influence the gathering of dogs in a previous study in Ayutthaya, Thailand [49]. The networks for the dogs observed on weekends and weekdays were created to demonstrate the difference in dog-to-dog contacts occurring, as suspected to be influenced by human activity. Since the caretakers often feed dogs from Monday to Friday, we expected to see higher connectedness in the weekday network. However, the size of the network was the only difference observed between the weekday and weekend networks. Since, there was no evidence that the dogs observed during weekdays and weekends were distinct from one another and the overall, weekday and weekend dogto-dog networks showed similar characteristics including high clustering, short average path length and high fragmentation, the understanding of disease transmission in this particular dog population should be based on the overall network.

Fragmentation of a network reflects the proportion of nodes that are not joined by direct or indirect edges. The observed dog-to-dog networks had high fragmentation, leading to a large number of components in the network. A component is defined as a group of nodes connected by at least one edge. The largest component was used to calculate the nBetweenness and nCloseness since all the nodes were not connected within the network. The largest component in the overall network had 117 connected nodes. For exploratory purposes, targeted removal of cut-points in the largest component could create up to 12 smaller components (including isolates). Roaming range was not explicitly measured and was beyond the scope of this study. In the study area, a dog could hypothetically roam across the entire campus area, although previous studies $[32,50]$ and comments from feeders suggest that they are sedentary and have a high affinity to certain location. Because the spatial proximity enables them to spread disease, we included all dogs on campus, despite their affiliation with a community. Theoretically, identifying the dogs that act as the bridge between subcomponents (called "communities") and severing the edges between subcomponents of dogs could decrease the risk of disease spread between clusters and, therefore, decrease the speed of disease spread in the event of an outbreak. In terms of 
rabies control, vaccinating the dog or capturing and restricting its' movement has the potential to prevent the infection and stop the spread of disease. The ability to identify and prioritize high-risk dogs for intervention will be useful in developing targeted rabies control strategies.

Betweenness is measured as the number of shortest paths that pass through the node. Dogs with high betweenness would likely be the dogs that create edges between components and may have considerable influence on the network. In our study, the same group of dogs was found repeatedly in the same area. Therefore, it was also highly suggestive that the dogs with high betweenness roamed between multiple observed locations. In our study, the dog with edges to the most polygons (dog number 42) was identified as an intact female who exhibited friendly behavior to humans. There was no documented information on her reproductive status, for example, whether she was in estrus, which could have influenced her roaming patterns $[27,48,51]$. She was observed in four polygons (all categorized as urban) in total. During observations, this dog was observed with up to six other dogs and in total, she had contacts with 14 other dogs during the study. Dog number 42 was also included in the main component and her nBetweenness was high (14.89, while mean betweenness $=3.95$ ). Dogs with high nBetweenness have great potential for disease spread. This is even more evident when roaming behavior (quantified by the number of polygon degrees computed from the two-mode network) is considered, as demonstrated in our study.

The degree calculated from the dog-to-polygon two-mode network in our study was highly suggestive of affinity of a dog to a place $[47,52]$ as most of the studied dogs were repeatedly seen in the same polygon. Interestingly, there was no statistical difference in the number of dogs between urban and rural polygons. This suggests that other factors could likely play a more important role in determining the carrying capacity of dogs in each area. For instance, areas supervised by at least one human feeder could have more plentiful resources, sustaining and attracting a larger number of dogs. Further studies should be conducted in order to determine the important factors involved in the carrying capacity of free-roaming dogs in Thailand.

Previous studies $[44,53]$ have demonstrated the benefits of using network models and contact network epidemiology to provide meaningful insights into the complex interaction between disease transmission and the contact patterns of free-roaming dogs. Estimating the speed and extent of the spread of a certain disease, such as rabies, and identifying a possible mitigation strategy specific to the network can result in developing practical control measures for the specific disease.

Considering the observational methods used in our study, the cross-sectional nature of the data collection only described a snapshot of potential contacts, rather than a continuous monitoring of dogs' movements and contacts such as demonstrated in studies obtained using GPS tracking. Moreover, observational methods required the observer to be in close proximity with the dogs, which could have affected their behavior. However, the results produced from this study prove observational methods to be a simple and cost-effective alternative that can be applied in rural and resource-limited settings, where network data could be extremely valuable. Although the data used in this study were sufficient to create the networks of interest, a longitudinal study that can capture dog movements and their contacts continuously for the entire study period would likely provide additional valuable insights. Further validation of the cross-sectional design used in this study against concurrent longitudinal data will be useful in determining the similarities between networks generated from cross-sectional and longitudinal data.

The socio-ecology of free-roaming dogs is being increasingly studied in Thailand. In comparison, many common behavioral traits are shared with free-roaming dogs from other countries, such as affinity to a certain place with varying human familiarity [2,52]. The abundance of food provided by human feeders has created a distinct environment for the free-roaming dogs in Thailand. Previous studies have reported most of the dogs encountered appear to be in good body condition, very few dogs were emaciated $[50,51,54]$ 
Furthermore, a study in Phitsanulok, Thailand, described a cluster of free-roaming dogs co-existing with a group of labor workers, similar to what we observed in this study [50]. In our study, we observed that most dogs on campus receive food from a caretaker. This is commonly observed in Thai communities. Moreover, two patterns of dog-caretaker associations that were previously observed in Prachuap Khiri Khan, Thailand [55] were also observed in this study. The dogs that are friendly and could be easily restrained by the caretaker and the dogs that do not tolerate any kind of human contact. These two distinct types of dogs are frequently observed in other rural communities in Thailand. Even though our study was based inside a university campus, we expect to see similar contact patterns and network characteristics in free-roaming dog populations with a caretaker associated with places with regular working hours.

\section{Conclusions}

This study described the network of community dogs on a university campus in Thailand. The free-roaming dog network described in this study had a small-world network characteristics with a right-skewed degree distribution, consistent with previously reported free-roaming dog networks in other countries. The high betweenness and high normalized degree measures were used in identifying dogs that had a higher likelihood of transmitting disease. The contact patterns of dogs on the campus were largely influenced by human activity. The weekend network showed that dogs tend to form larger groups during weekends, in the absence of humans. The two-mode dog-to-polygon network revealed that most dogs had an affinity to a place. The number of dogs observed in an area was not different between urban and rural areas likely because there were caretakers providing food in both types of areas. This study was conducted using a cross-sectional design; further studies should be conducted in order to determine the similarities of the networks created using a longitudinal study design. The network information acquired from this study can be further used in network modeling and designing targeted disease control programs.

Supplementary Materials: The following are available online at https:/ /www.mdpi.com/article/ 10.3390/vetsci8120299/s1, Figure S1: Cut-points and subcomponents identified within the main component of the overall dog-to-dog network, Table S1: Summary of all permutation-based statistical analyses performed, Figure S2: Plots showing the number of dogs from the overall, weekday and weekend dog-to-dog networks stratified by behavioral response to an unfamiliar human.

Author Contributions: Conceptualization, S.K. and W.P.; methodology, T.K., S.K., W.P. and K.K.T.; formal analysis, T.K. and K.K.T.; investigation, T.K.; writing-original draft preparation, T.K.; writing—-review and editing, T.K., S.K., W.P. and K.K.T.; visualization, T.K.; supervision, S.K., W.P. and K.K.T.; funding acquisition, T.K., S.K. and W.P. All authors have read and agreed to the published version of the manuscript.

Funding: This research was partially supported by the Saving Animal and Human Lives from Rabies Project following the Determination of HRH Princess Chulabhorn. TK received the research assistance scholarship for graduate students from the Faculty of Veterinary Medicine, Kasetsart University while conducting the research in Thailand. TK also received the Canada-ASEAN Scholarships and Educational Exchanges for Development (SEED) scholarship from Global Affairs Canada which provided the opportunity to partially conduct the research at the Atlantic Veterinary College, University of Prince Edward Island in Canada.

Institutional Review Board Statement: The study was conducted according to the guidelines of the Declaration of Helsinki and approved by the Institutional Review Board (or Ethics Committee) of Institutional Animal Care and Use Committee (IACUC), Kasetsart University (ID: ACKU64-VET-058 received on 9 August 2021).

Informed Consent Statement: Not applicable.

Data Availability Statement: The data presented in this study are available in this article. 
Acknowledgments: The authors would like to acknowledge Kasetsart University, Kamphaeng Saen campus for the permission to conduct the study.

Conflicts of Interest: The authors declare no conflict of interest. The funders had no role in the design of the study; in the collection, analyses or interpretation of data; in the writing of the manuscript, or in the decision to publish the results.

\section{Appendix A}

Assumed human activity, for each of the polygon, was ranked from low ( $<20$ persons), moderate (20-100 persons) and high (over 100 persons), based on Kasetsart University's report of personnel in 2019 [56] and observations during data collection. Human activities for areas not covered in the report, including cafeterias and lecture halls, were estimated from visual observations and categorized according to the criteria described above. A satellite photograph [57] of the campus was used to assess the occupancy of human-made structures within the polygon. According to a satellite image of the campus, polygons were ranked based on the proportion of area occupied by man-made structures within the polygon. It ranged from high $(>0.5)$, moderate $(0.25-0.5)$ and low $(<0.25)$.

\section{References}

1. Boitani, L.; Ciucci, P. Comparative social ecology of feral dogs and wolves. Ethol. Ecol. Evol. 1995, 7, 49-72. [CrossRef]

2. Ortolani, A.; Vernooij, H.; Coppinger, R. Ethiopian village dogs: Behavioural responses to a stranger's approach. Appl. Anim. Behav. Sci. 2009, 119, 210-218. [CrossRef]

3. World Health Organization. Report of WHO Consultation on Dog Ecology Studies Related to Rabies Control; World Health Organization: Geneva, Switzerland, 1988.

4. Jackman, J.; Rowan, A. Free-Roaming Dogs in Developing Countries: The Benefits of Capture, Neuter, and Return Programs; Humane Society Press: Washington, DC, USA, 2007; pp. 55-78.

5. Kongkaew, W.; Coleman, P.; Pfeiffer, D.U.; Antarasena, C.; Thiptara, A. Vaccination coverage and epidemiological parameters of the owned-dog population in Thungsong District, Thailand. Prev. Vet. Med. 2004, 65, 105-115. [CrossRef] [PubMed]

6. Premashthira, S.; Suwanpakdee, S.; Thanapongtharm, W.; Sagarasaeranee, O.; Thichumpa, W.; Sararat, C.; Wiratsudakul, A. The Impact of Socioeconomic Factors on Knowledge, Attitudes, and Practices of Dog Owners on Dog Rabies Control in Thailand. Front. Vet. Sci. 2021, 8, 699352. [CrossRef]

7. Yen, S.C.; Ju, Y.T.; Shaner, P.L.; Chen, H.L. Spatial and temporal relationship between native mammals and free-roaming dogs in a protected area surrounded by a metropolis. Sci. Rep. 2019, 9, 8161. [CrossRef] [PubMed]

8. World Health Organization. WHO Expert Committee on Rabies [Meeting Held in Geneva from 20 to 27 September 1983]; Seventh Report; World Health Organization: Geneva, Switzerland, 1984.

9. World Health Organization. Oral Vaccination of Dogs against Rabies: Guidance for Research on Oral Rabies Vaccines and Field Application of Oral Vaccination of Dogs against Rabies; World Health Organization: Geneva, Switzerland, 2007.

10. Department of Livestock Development. Thai Rabies Net. Available online: http://www.thairabies.net/trn/ (accessed on 27 September 2021).

11. Kasempimolporn, S.; Sichanasai, B.; Saengseesom, W.; Puempumpanich, S.; Chatraporn, S.; Sitprija, V. Prevalence of rabies virus infection and rabies antibody in stray dogs: A survey in Bangkok, Thailand. Prev. Vet. Med. 2007, 78, 325-332. [CrossRef]

12. Chanachai, K.; Wongphruksasoong, V.; Vos, A.; Leelahapongsathon, K.; Tangwangvivat, R.; Sagarasaeranee, O.; Lekcharoen, P.; Trinuson, P.; Kasemsuwan, S. Feasibility and Effectiveness Studies with Oral Vaccination of Free-Roaming Dogs against Rabies in Thailand. Viruses 2021, 13, 571. [CrossRef]

13. Christakis, N.A.; Fowler, J.H. The Spread of Obesity in a Large Social Network over 32 Years. N. Engl. J. Med. 2007, 357, 370-379. [CrossRef]

14. Malik, H.A.M.; Mahesar, A.W.; Abid, F.; Waqas, A.; Wahiddin, M.R. Two-mode network modeling and analysis of dengue epidemic behavior in Gombak, Malaysia. Appl. Math. Model. 2017, 43, 207-220. [CrossRef]

15. Godfrey, S.S.; Ansari, T.H.; Gardner, M.G.; Farine, D.R.; Bull, C.M. A contact-based social network of lizards is defined by low genetic relatedness among strongly connected individuals. Anim. Behav. 2014, 97, 35-43. [CrossRef]

16. Lusseau, D.; Newman, M.E. Identifying the role that animals play in their social networks. Proc. Biol. Sci. 2004, 271 (Suppl. 6), S477-S481. [CrossRef]

17. Flack, J.C.; Girvan, M.; de Waal, F.B.; Krakauer, D.C. Policing stabilizes construction of social niches in primates. Nature 2006, 439, 426-429. [CrossRef]

18. Ortiz-Pelaez, A.; Pfeiffer, D.U.; Soares-Magalhaes, R.J.; Guitian, F.J. Use of social network analysis to characterize the pattern of animal movements in the initial phases of the 2001 foot and mouth disease (FMD) epidemic in the UK. Prev. Vet. Med. 2006, 76, 40-55. [CrossRef] 
19. Dube, C.; Ribble, C.; Kelton, D.; McNab, B. Comparing network analysis measures to determine potential epidemic size of highly contagious exotic diseases in fragmented monthly networks of dairy cattle movements in Ontario, Canada. Transbound. Emerg. Dis. 2008, 55, 382-392. [CrossRef] [PubMed]

20. Rautureau, S.; Dufour, B.; Durand, B. Structural vulnerability of the French swine industry trade network to the spread of infectious diseases. Animal 2012, 6, 1152-1162. [CrossRef] [PubMed]

21. Lichoti, J.K.; Davies, J.; Kitala, P.M.; Githigia, S.M.; Okoth, E.; Maru, Y.; Bukachi, S.A.; Bishop, R.P. Social network analysis provides insights into African swine fever epidemiology. Prev. Vet. Med. 2016, 126, 1-10. [CrossRef]

22. Thakur, K.K.; Revie, C.W.; Hurnik, D.; Poljak, Z.; Sanchez, J. Analysis of Swine Movement in Four Canadian Regions: Network Structure and Implications for Disease Spread. Transbound. Emerg. Dis. 2016, 63, e14-e26. [CrossRef] [PubMed]

23. Dougherty, E.R.; Seidel, D.P.; Carlson, C.J.; Spiegel, O.; Getz, W.M. Going through the motions: Incorporating movement analyses into disease research. Ecol. Lett. 2018, 21, 588-604. [CrossRef]

24. Robitaille, A.L.; Webber, Q.M.R.; Vander Wal, E. Conducting social network analysis with animal telemetry data: Applications and methods using spatsoc. Methods Ecol. Evol. 2019, 10, 1203-1211. [CrossRef]

25. Silk, M.J.; Croft, D.P.; Delahay, R.J.; Hodgson, D.J.; Boots, M.; Weber, N.; McDonald, R.A. Using Social Network Measures in Wildlife Disease Ecology, Epidemiology, and Management. BioScience 2017, 67, 245-257. [CrossRef]

26. Westgarth, C.; Gaskell, R.M.; Pinchbeck, G.L.; Bradshaw, J.W.; Dawson, S.; Christley, R.M. Walking the dog: Exploration of the contact networks between dogs in a community. Epidemiol. Infect. 2009, 137, 1169-1178. [CrossRef]

27. Brookes, V.J.; VanderWaal, K.; Ward, M.P. The social networks of free-roaming domestic dogs in island communities in the Torres Strait, Australia. Prev. Vet. Med. 2020, 181, 104534. [CrossRef]

28. Warembourg, C.; Fournié, G.; Abakar, M.F.; Alvarez, D.; Berger-González, M.; Odoch, T.; Wera, E.; Alobo, G.; Carvallo, E.T.L.; Bal, V.D.; et al. Predictors of free-roaming domestic dogs' contact network centrality and their relevance for rabies control. Sci. Rep. 2021, 11, 12898. [CrossRef]

29. Wilson-Aggarwal, J.K.; Ozella, L.; Tizzoni, M.; Cattuto, C.; Swan, G.J.F.; Moundai, T.; Silk, M.J.; Zingeser, J.A.; McDonald, R.A. High-resolution contact networks of free-ranging domestic dogs Canis familiaris and implications for transmission of infection. PLoS Negl. Trop. Dis. 2019, 13, e0007565. [CrossRef]

30. Brookes, V.J.; Dürr, S.; Ward, M.P. Rabies-induced behavioural changes are key to rabies persistence in dog populations: Investigation using a network-based model. PLoS Negl. Trop. Dis. 2019, 13, e0007739. [CrossRef] [PubMed]

31. Kasetsart University Kamphaeng Saen Campus. ABOUT KU KPS. Available online: https://kps.ku.ac.th/v8/index.php/en/ about-us/about-us-ku-kps (accessed on 7 April 2021).

32. Phokham, K.; Therakul, N.; Eurwilaichit, A.; Boonkasemsanti, A.; Leelahapongsathon, K.; Kasemsuwan, S. A comparison of Roaming-dog population estimation method in Kasetsart University, Kamphaeng Saen Campus, Nakhon Pathom. In Proceedings of the 29th Case Study Conference of 6th Year Students, Faculty of Veterinary Medicine, Kasetsart University, Nakhon Pathom, Thailand, 14-15 March 2020.

33. Gohil, R.; Sharma, S.; Sachdeva, S.; Gupta, S.; Dhillon, M. EpiCollect 5: A Free, Fully Customizable Mobile-Based Application for Data Collection in Clinical Research. J. Postgrad. Med. Educ. Res. 2020, 54. [CrossRef]

34. Aanensen, D.M.; Huntley, D.M.; Feil, E.J.; al-Own, F.A.; Spratt, B.G. EpiCollect: Linking Smartphones to Web Applications for Epidemiology, Ecology and Community Data Collection. PLoS ONE 2009, 4, e6968. [CrossRef] [PubMed]

35. R Core Team, R. A Language and Environment for Statistical Computing; R Core Team: Vienna, Austria, 2020.

36. QGIS.org. QGIS Geographic Information System. 2021. Available online: https:// qgis.org/en/site/ (accessed on 23 October 2020).

37. Hugentobler, M. Quantum GIS. In Encyclopedia of GIS; Shekhar, S., Xiong, H., Eds.; Springer: Boston, MA, USA, 2008 ; pp. 935-939.

38. Borgatti, S.P.; Everett, M.G.; Freeman, L.C. UCINET 6 for Windows: Software for Social Network Analysis; Analytic Technologies: Harvard, MA, USA, 2002.

39. Bastian, M.; Heymann, S.; Jacomy, M. Gephi: An Open Source Software for Exploring and Manipulating Networks. In Proceedings of the International AAAI Conference on Web and Social Media, San Jose, CA, USA, 17-20 May 2009; Volume 3, pp. 361-362.

40. Borgatti, S.P. NetDraw: Graph.Visualization Software. 2002. Available online: https://sites.google.com/site/netdrawsoftware/ (accessed on 5 February 2021).

41. Humphries, M.D.; Gurney, K. Network 'Small-World-Ness': A Quantitative Method for Determining Canonical Network Equivalence. PLOS ONE 2008, 3, e0002051. [CrossRef]

42. Humphries, M.D.; Gurney, K.; Prescott, T.J. The brainstem reticular formation is a small-world, not scale-free, network. Proc. Biol. Sci. 2006, 273, 503-511. [CrossRef] [PubMed]

43. Montoya, J.M.; SolÉ, R.V. Small World Patterns in Food Webs. J. Theor. Biol. 2002, 214, 405-412. [CrossRef]

44. Craft, M.E.; Volz, E.; Packer, C.; Meyers, L.A. Disease transmission in territorial populations: The small-world network of Serengeti lions. J. R. Soc. Interface 2011, 8, 776-786. [CrossRef]

45. Watts, D.J.; Strogatz, S.H. Collective dynamics of 'small-world' networks. Nature 1998, 393, 440-442. [CrossRef] [PubMed]

46. Daniels, T.J. The social organization of free-ranging urban dogs. I. Non-estrous social behavior. Appl. Anim. Ethol. 1983, 10, 341-363. [CrossRef]

47. Belo, V.S.; Werneck, G.L.; da Silva, E.S.; Barbosa, D.S.; Struchiner, C.J. Population Estimation Methods for Free-Ranging Dogs: A Systematic Review. PLoS ONE 2015, 10, e0144830. [CrossRef] 
48. Hudson, E.G.; Brookes, V.J.; Durr, S.; Ward, M.P. Domestic dog roaming patterns in remote northern Australian indigenous communities and implications for disease modelling. Prev. Vet. Med. 2017, 146, 52-60. [CrossRef]

49. Sangarun, K.; Phimpraphai, W.; Leelahapongsathon, K.; Binot, A.; Kasemsuwan, S. Estimation of Dogs by Photogenic CaptureRecapture Technique in Various Sites at Chiang Rak Noi Subdistrict, Ayutthaya Province, Thailand. In Proceedings of the 5th Food Safety and Zoonoses Symposium for Asia Pacific, Chiang Mai, Thailand, 6-7 July 2018; pp. 50-54.

50. Sirasoonthorn, P.; Suesat, W.; Thongyuan, S.; Pinyopummintr, T. Social Interaction between Labors and Stray Dogs, and Information of Rabies in Urban Temporary Construction Sides, Phitsanulok Province. In Proceedings of the 14th Ubon Ratchathani University National Research Conference, Ubon Ratchathani, Thailand, 3-4 September 2020.

51. Maher, E.K.; Ward, M.P.; Brookes, V.J. Investigation of the temporal roaming behaviour of free-roaming domestic dogs in Indigenous communities in northern Australia to inform rabies incursion preparedness. Sci. Rep. 2019, 9, 14893. [CrossRef]

52. Miternique, H.C.; Gaunet, F. Coexistence of Diversified Dog Socialities and Territorialities in the City of Concepción, Chile. Animals 2020, 10, 298. [CrossRef]

53. Pourbohloul, B.; Meyers, L.A.; Skowronski, D.M.; Krajden, M.; Patrick, D.M.; Brunham, R.C. Modeling control strategies of respiratory pathogens. Emerg. Infect. Dis. 2005, 11, 1249-1256. [CrossRef]

54. Kasemsuwan, S.; Chanachai, K.; Pinyopummintr, T.; Leelalapongsathon, K.; Sujit, K.; Vos, A. Field Studies Evaluating Bait Acceptance and Handling by Free-Roaming Dogs in Thailand. Vet. Sci. 2018, 5, 47. [CrossRef]

55. Leelahapongsathon, K.; Horpiencharoen, W.; Thongyuan, S.; Kasemsuwan, S.; Pinyopummintr, T. Rapid community dog assessment in rabies endemic area. Int. J. Infect. Dis. 2019, 79, 35. [CrossRef]

56. Kasetsart University Kamphaeng Saen Campus Information and Technology Center. Kasetsart University Kamphaeng Saen Campus Personnel Database. Available online: https:/ / kpsoffice.kps.ku.ac.th/index.php (accessed on 14 March 2020).

57. Google. Google Maps. Available online: https:/ / www.google.com/maps (accessed on 18 June 2020). 